\title{
POM.gpu-v1.0: a GPU-based Princeton Ocean Model
}

\author{
S. Xu ${ }^{1}$, X. Huang ${ }^{1}$, L.-Y. Oey ${ }^{2,3}$, F. Xu ${ }^{1}$, H. Fu ${ }^{1}$, Y. Zhang ${ }^{1}$, and G. Yang ${ }^{1}$ \\ ${ }^{1}$ Ministry of Education Key Laboratory for Earth System Modeling, Center for Earth System Science, Tsinghua University, \\ 100084, and Joint Center for Global Change Studies, Beijing, 100875, China \\ ${ }^{2}$ Institute of Hydrological \& Oceanic Sciences, National Central University, Jhongli, Taiwan \\ ${ }^{3}$ Program in Atmospheric \& Oceanic Sciences, Princeton University, Princeton, New Jersey, USA
}

Correspondence to: X. Huang (hxm@tsinghua.edu.cn)

Received: 13 October 2014 - Published in Geosci. Model Dev. Discuss.: 17 November 2014

Revised: 10 August 2015 - Accepted: 19 August 2015 - Published: 9 September 2015

\begin{abstract}
Graphics processing units (GPUs) are an attractive solution in many scientific applications due to their high performance. However, most existing GPU conversions of climate models use GPUs for only a few computationally intensive regions. In the present study, we redesign the mpiPOM (a parallel version of the Princeton Ocean Model) with GPUs. Specifically, we first convert the model from its original Fortran form to a new Compute Unified Device Architecture C (CUDA-C) code, then we optimize the code on each of the GPUs, the communications between the GPUs, and the I/O between the GPUs and the central processing units (CPUs). We show that the performance of the new model on a workstation containing four GPUs is comparable to that on a powerful cluster with 408 standard CPU cores, and it reduces the energy consumption by a factor of 6.8 .
\end{abstract}

\section{Introduction}

High-resolution atmospheric, oceanic and climate modellings remain significant scientific and engineering challenges because of the enormous computing, communication, and storage requirements involved. Due to the rapid development of computer architecture, in particular the development of multi-core and many-core hardware, the computing power that can be applied to scientific problems has increased exponentially in recent decades. Parallel computing methods, such as the Message Passing Interface (MPI, Gropp et al., 1999) and Open Multi-Processing (OpenMP, Chapman et al., 2008), have been widely used to support the parallelization of climate models. However, supercomputers are becoming increasingly heterogeneous, involving devices such as the GPU and the Intel Many Integrated Core (Intel MIC), and new approaches are required to effectively utilize the new hardware.

In recent years, a number of scientific codes have been ported to the GPU as shown in Table 1. Most existing GPU acceleration codes for climate models are only operating on certain hot spots of the program, leaving a significant portion of the program still running on CPUs. The speed of some subroutines reported in the Weather Research and Forecast (WRF) (Michalakes and Vachharajani, 2008) and WRF-Chem (Linford et al., 2009) is improved by a factor of approximately 8 , whereas the whole model achieves limited speedup because of partial porting. The speed of POP (Zhenya et al., 2010) is improved by a factor of only 2.2 because the model only accelerated a number of loop structures using the OpenACC Application Programming Interface (OpenACC API). The speed of COSMO (Leutwyler et al., 2014), NIM (Govett et al., 2010) and ASUCA (Shimokawabe et al., 2010) are greatly improved by multiple GPUs. We believe that the elaborate optimization of the memory access of each GPU and the communication between GPUs can further accelerate these models.

The objective of our study was to shorten the computation time of the Princeton Ocean Model (POM) by parallelizing its existing model structures using the GPU. Taking the parallel version of the Princeton Ocean Model (mpiPOM), we demonstrate how to code an ocean model so that it runs efficiently on GPU architecture. We first convert the mpiPOM from its original Fortran version into a new Compute Unified Device Architecture C (CUDA-C) version, POM.gpuv1.0. CUDA-C is the dominant programming language for GPUs. We then optimize the code on each of the GPUs, the communications between the GPUs, and the I/O between 
Table 1. Existing GPU porting work in climate fields. The speedups are normalized to one CPU core.

\begin{tabular}{lllc}
\hline Model name & Model description & Porting modules to GPU & Speedup \\
\hline WRF & Weather Research and Forecasting & WSM5 microphysics & 8 \\
WRF-Chem & WRF chemical & Chemical kinetics kernel & 8.5 \\
POP & Parallel Ocean Program & Loop structures & 2.2 \\
COSMO & Consortium for Small-scale Modeling & Dynamical core & 22.7 \\
NIM & Nonhydrostatic Icosahedral Model & Dynamical core & 34 \\
ASUCA & Nonhydrostatic weather model & Dynamical core and physical & 80 \\
\hline
\end{tabular}

GPUs and the CPUs to further improve the performance of POM.gpu.

To understand the accuracy, performance and scalability of the POM.gpu code, we customized a workstation with four Nvidia K20X GPUs. The results show that the performance of POM.gpu running on this workstation is comparable to that on a powerful cluster with 408 standard CPU cores.

This paper is organized as follows. In Sect. 2, we review the mpiPOM model. In Sect. 3, we briefly introduce the GPU computing model. In Sect. 4, we present the detailed optimization techniques. In Sect. 5, we report on the correctness, performance and scalability of the model. We present the code availability in Sect. 6 and conclude our work in Sect. 7.

\section{The mpiPOM}

The mpiPOM is a parallel version of the POM. It retains most of the physics of the original POM (Blumberg and Mellor, 1983, 1987; Oey et al., 1985a, b, c; Oey and Chen, 1992a, b) and includes satellite and drifter assimilation schemes from the Princeton Regional Ocean Forecast System (Oey, 2005; Lin et al., 2006; Yin and Oey, 2007), stokes drift and waveenhanced mixing (Oey et al., 2013; Xu et al., 2013; Xu and Oey, 2014). The POM code was reorganized and the parallel MPI version was implemented by Jordi and Wang (2012) using a two-dimensional data decomposition of the horizontal domain. The MPI is a standard library for message passing and is widely used to develop parallel programs. The POM is a powerful ocean model that has been used in a wide range of applications: circulation and mixing processes in rivers, estuaries, shelves, slopes, lakes, semi-enclosed seas and open and global oceans. It is also at the core of various real-time ocean and hurricane forecasting systems, e.g. the Japanese coastal ocean and Kuroshio current (Miyazawa et al., 2009; Isobe et al., 2012; Varlamov et al., 2015), the Adriatic Sea Forecasting System (Zavatarelli and Pinardi, 2003), the Mediterranean Sea forecasting system (Korres et al., 2007), the GFDL Hurricane Prediction System (Kurihara et al., 1995, 1998), the US Hurricane Forecasting System (Gopalakrishnan et al., 2010, 2011), and the Advanced Taiwan Ocean Prediction (ATOP) system (Oey et al., 2013). Additionally, the model has been used to study various geophysical fluid dynamical processes (e.g. Allen and Newberger, 1996; New- berger and Allen, 2007a, b; Kagimoto and Yamagata, 1997; Guo et al., 2006; Oey et al., 2003; Zavatarelli and Mellor, 1995; Ezer and Mellor, 1992; Oey, 2005; Xu and Oey, 2011, 2014, 2015; Chang and Oey, 2014; Huang and Oey, 2015; Sun et al., 2014, 2015). For a more complete list, please visit the POM website (http://www.ccpo.odu.edu/POMWEB).

The mpiPOM experiment used in this paper is one of two that were designed and tested by Professor Oey and students; the codes and results are freely available at the FTP site (ftp:// profs.princeton.edu/leo/mpipom/atop/tests/). The reader can refer to Chapter 3 of the lecture notes (Oey, 2014) for more detail. The test case is a dam-break problem in which warm and cold waters are initially separated in the middle of a zonally periodic channel $(200 \mathrm{~km} \times 50 \mathrm{~km} \times 50 \mathrm{~m})$ on an $\mathrm{f}$ plane, with walls at the northern and southern boundaries. Geostrophic adjustment then ensues and baroclinic instability waves amplify and develop into finite-amplitude eddies in 10 20 days. The horizontal grid sizes are $1 \mathrm{~km}$ and there are 50 vertical sigma levels. Although the problem is a test case, the code is the full mpiPOM version used in the ATOP forecasting system.

The model solves the primitive equation under hydrostatic and Boussinesq approximations. In the horizontal, spatial derivatives are computed either using centered-space differencing or Smolarkiewicz's positive definite advection transport algorithm (Smolarkiewicz, 1984) on a staggered Arakawa C-grid; both schemes have been tested, but the latter is reported here. In the vertical, the mpiPOM supports terrain-following sigma coordinates and a fourth-order scheme option to reduce the internal pressure-gradient errors (Berntsen and Oey, 2010). The mpiPOM uses the timesplitting technique to separate the vertically integrated equations (external mode) from the vertical structure equations (internal mode). The external mode calculation is responsible for updating the surface elevation and vertically averaged velocities. The internal mode calculation updates the velocity, temperature and salinity, as well as the turbulence quantities. The three-dimensional internal mode and the twodimensional external mode are both integrated explicitly using a second-order leapfrog scheme. These two modules are the most computationally intensive kernels of the mpiPOM model. 
The main computational problem of the mpiPOM is memory bandwidth limited. To confirm this issue, we use the runtime performance API tool to estimate the floating point operation count and the memory access instruction count, as in Browne et al. (2000). The results reveal that the computational intensity, defined as floating point operations per byte transferred to or from memory, of the mpiPOM is approximately $1: 3.3$, whereas the computational intensity provided by a modern high-performance CPU (an Intel SandyBridge E5-2670) is 7.5:1. Many large arrays are mostly pulled from the main memory and there is poor data reuse in the mpiPOM. In addition, there are no obvious hot spot functions in the mpiPOM, and even the most time-consuming subroutine occupies only $20 \%$ of the total execution time. Therefore, porting a handful of subroutines to the GPU is not helpful in improving the model efficiency. This explains why we must port the entire program from the CPU to the GPU.

\section{GPU computing model overview}

Modern GPUs employ a stream-processing model with parallelism. Each GPU contains a number of stream multiprocessors (SMs). In this work, we carried out the conversion using four Nvidia K20X GPUs. Each K20X GPU contains 14 SMs and each SM has 192 single-precision processors and 64 additional processors for double precision. Although the computational capability of each processor is low, one GPU with thousands of processors can greatly boost the performance compared to the CPU. In computing, FLOPS (FLoating-point Operations Per Second) is a measure of computer performance. The theoretical peak performance of each K20X GPU is 3.93 teraFLOPS (TFLOPS, one trillion floating-point operations per second) for the single-precision floating-point calculations. In contrast, a single Intel SandyBridge E5-2670 CPU is only capable of 0.384 TFLOPS.

Each pair of GPUs shares 6 Gigabytes (GB) of memory, with the interface having a potential bandwidth of $250 \mathrm{~GB} \mathrm{~s}^{-1}$. Figure 3 illustrates the memory hierarchy of the K20X GPU. Each SM possesses some types of fast on-chip memory such as register, L1 cache, shared memory and readonly data cache. In GPUs, the register is the fastest memory, of which the size is 256 Kilobytes (KB) for each SM. The shared memory and the L1 cache use the common $64 \mathrm{~KB}$ space, which can be partitioned as $16 / 48 \mathrm{~KB}, 32 / 32 \mathrm{~KB}$ or $48 / 16 \mathrm{~KB}$. The $48 \mathrm{~KB}$ read-only data cache is useful for holding frequently used values that remain unchanged during each stage of the processing.

There are three widely used methods for porting a program to GPUs. The first method uses drop-in libraries provided by CUDA to replace the existing code, as in Siewertsen et al. (2013). The second method uses the OpenACC directive as hints in the original CPU code as in Zhenya et al. (2010). The last method is the most complex but also the most effective; it

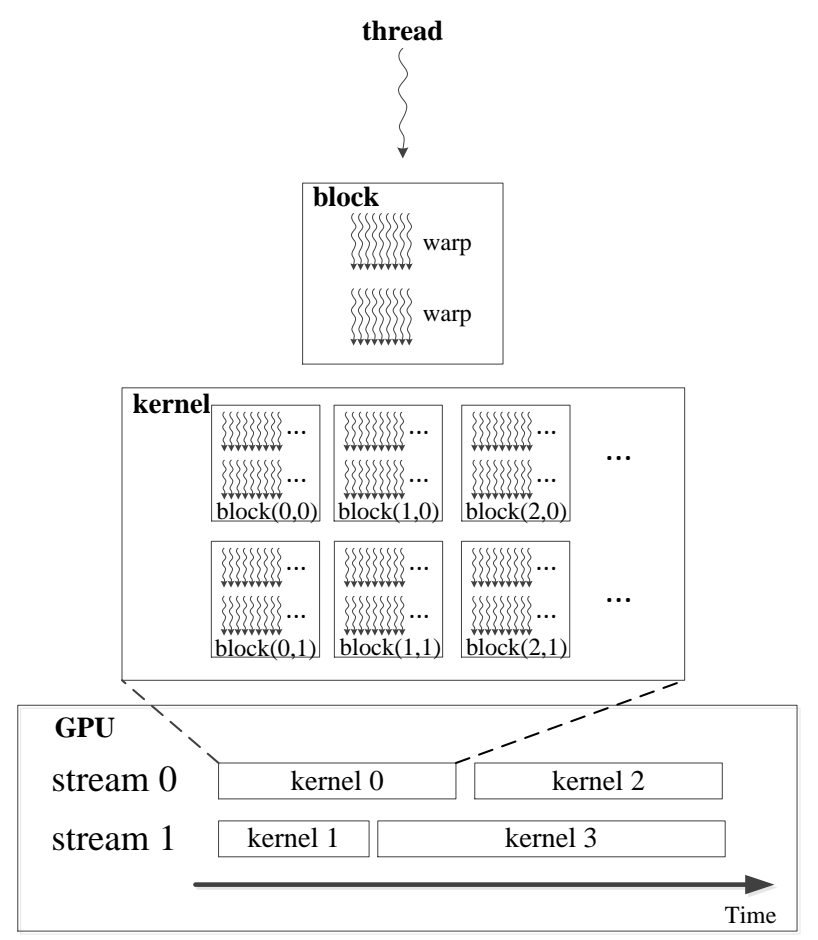

Figure 1. The hierarchy of stream, kernel, block, warp and thread.

involves rewriting the entire program using low-level CUDA subroutines.

In CUDA terminology, a kernel is a single section of code or subroutine running on the GPU. The underlying code in a kernel is split into a series of threads, each of which deals with different data. These threads are grouped into equal-size thread blocks that can be executed independently. A thread block is further divided into warps as basic scheduled units. A warp consists of 32 consecutive threads that execute the same instruction simultaneously. Each kernel and data transfer command in CUDA has an optional parameter, "stream ID". If the stream ID is set in code, commands belonging to different streams can be executed concurrently. A stream in CUDA is a sequence of commands executed in order. Different streams can execute concurrently with different priorities. Figure 1 illustrates the hierarchy of these terms.

At present, CUDA compilers are available for $\mathrm{C}$ and Fortran. Although CUDA-Fortran has been available since 2009 and would involve less modification of the mpiPOM code, we chose CUDA-C to convert the POM.gpu-v1.0 because of the following reasons: (1) CUDA-C is free of charge; (2) previous work (Henderson et al., 2011) has shown that the CUDA-Fortran compiler did not perform as well as the CUDA-C version for some of the kernels during the porting of NIM; (3) the read-only data cache is not supported by CUDA-Fortran, which is the key optimization of Sect. 4.1.2; and (4) we have many previous optimization experiences with CUDA-C. 


\section{Full GPU acceleration of the mpiPOM}

Figure 2 is a flowchart illustrating the structure of the POM.gpu. The main difference between the mpiPOM and the POM.gpu is that the CPU in the POM.gpu is only responsible for the initializing and the output work. The POM.gpu begins by initializing the relevant arrays on the CPU and then copies data from the CPU to the GPU. The GPU then performs all of the model computations. Outputs such as velocity and sea-surface height ( $\mathrm{SSH}$ ) are copied back to the CPU and are then written to the disk at a user-specified time interval.

In the following sections, we introduce the optimizations of the POM.gpu by computation, communication and I / O aspects individually.

For the individual GPUs, we concentrate on memory access optimization by making better use of caches in the GPU memory hierarchy. This involves using read-only data cache, local memory blocking, loop fusion and function fusion, and disabling error-correcting code memory. The test results demonstrate that a single GPU can run the model almost 100 times faster than a single CPU core.

In terms of communication, we overlapped the sending of boundary data between the GPUs with the main computation. Data are also sent directly between the GPUs, bypassing the CPU.

In terms of I / O, we launched extra MPI processes on the main CPU to output the data. These MPI processes are divided into two categories, the computation processes and the $\mathrm{I} / \mathrm{O}$ processes. The computation processes are responsible for launching kernels into GPUs and the I / O processes are responsible for copying data back from the GPUs and for writing to disks. The computation processes and the I/O processes can execute simultaneously to save output time.

\subsection{Computational optimizations in a single GPU}

Managing the significant performance difference between global memory and on-chip fast memory is the primary concern for GPU computing. The ratio of bandwidth between global memory and shared memory is approximately $1: 10$. Therefore, data reuse in an on-chip cache always needs to be seriously considered. As shown on the right side of Fig. 3, we propose two classes of optimization, including the standard optimization of fusion and the special optimization of the GPU, to better utilize the fast registers and caches.

\subsubsection{Standard optimizations of fusion}

Fusion optimization in the POM.gpu code includes loop fusion and function fusion. The loop fusion merges several loops into one loop and the function fusion merges several subroutines into one subroutine.

Loop fusion is an effective method to store scalar variables in registers for data reuse. As shown in Fig. 4, if the variable

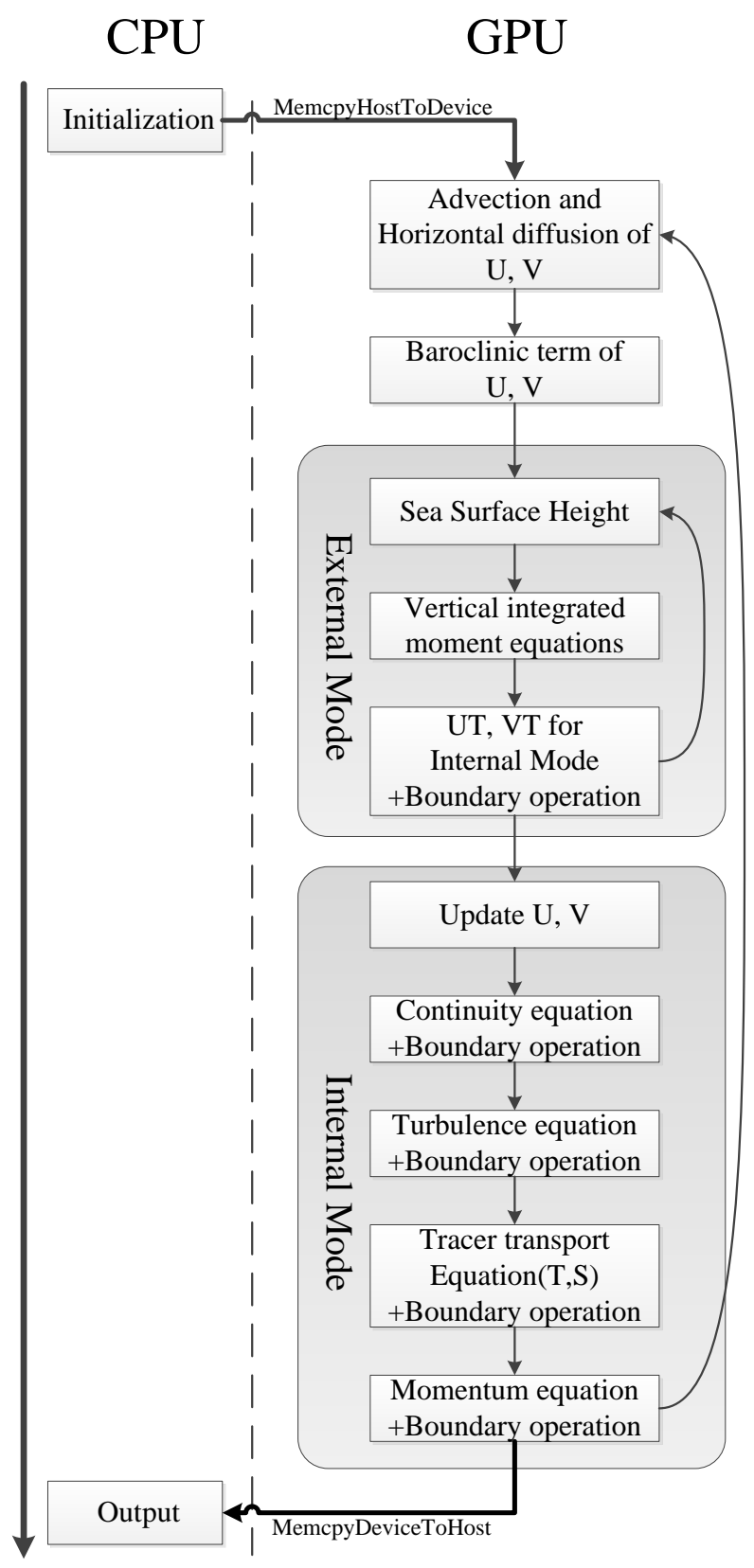

Figure 2. POM.gpu flowchart.

$\operatorname{drhox}(k, j, i)$ is read several times in multiple loops, we can fuse these loops into one. Therefore, $\operatorname{drhox}(k, j, i)$ will first be read from the global memory and then repeatedly read from a register. For instance, for the $p r o f q$ kernel optimized with loop fusion, the device memory transactions decrease by $57 \%$, and the running speed of this kernel is improved by $28.6 \%$. The loop fusion optimization can also be applied in a number of mpiPOM subroutines.

Similar to loop fusion, we can also merge functions in which the same arrays are accessed. For example, the $a d v v$ and $a d v u$ functions in the mpiPOM code are used to calculate 


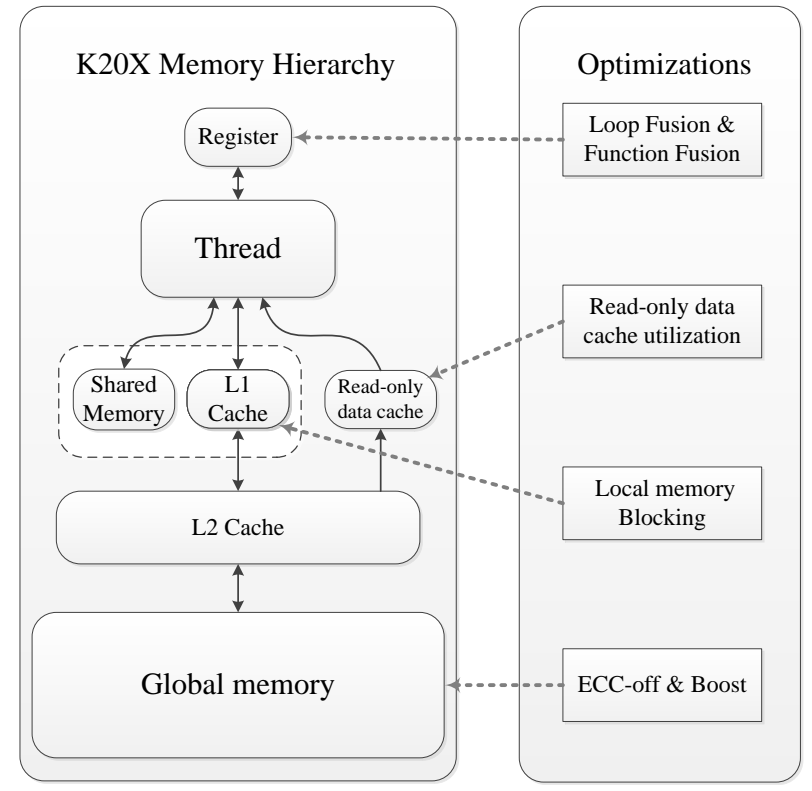

Figure 3. The memory hierarchy of the K20X GPU and the relationships with each optimization.

the advection terms in horizontal directions, respectively. After merging them into one subroutine, the redundant memory access is avoided. The function fusion can also be applied in which one function is called several times to calculate different tracers. The proft function in the mpiPOM code is called twice - one for temperature and one for salinity. Their computing formulas are similar and some common arrays are accessed. After function fusion, the running speed of the proft kernel is improved by $28.8 \%$.

\subsubsection{Special optimizations of the GPU}

Our special optimizations mainly focus on the improved utilization of the read-only data cache and the L1 cache on the GPU. It is useful to alleviate the bottleneck of memory bandwidth that is limited by using these fast on-chip caches.

There is a $48 \mathrm{~KB}$ read-only data cache in the K20X GPU. We can automatically use this as long as the read-only condition is met. In the POM.gpu, we simply add const _restrict_qualifiers into the parameter pointers to explicitly direct the compiler to implement the optimization. As an example, consider the calculations of advection and the horizontal diffusion terms. Because mpiPOM adopts the Arakawa Cgrid, in the horizontal plane, updating the temperature $(T)$ requires the velocity of longitude $(u)$, the velocity of latitude $(v)$ and the horizontal kinematic viscosity (aam) on the neighbouring grid points. In one kernel, the $u$ and $v$ arrays are accessed twice, and the aam array is accessed four times. After using the read-only data cache to improve the data locality, the running speed of this kernel is improved by $18.8 \%$.
To reuse the data in each thread, we use local memory blocking to pull the data from global memory to the L1 cache. In this method, a small subset of a data set is loaded into the fast on-chip memory and then the small data block is repeatedly accessed by the program. This method is helpful in reducing the need to access the off-chip with high latency memory. In the subroutines of the vertical diffusion and source/sink terms, the chasing method is used to solve a tridiagonal matrix along the vertical direction for each grid point individually. Each thread only accesses its own tiles of row transformation coefficients. As shown in Fig. 5, the arrays are accessed twice within one thread, one from the surface $(k=0)$ to the bottom $(k=n z-1)$ and another from the bottom $(k=n z-1)$ to the surface $(k=0)$. After blocking the vertical direction arrays in local memory, the L1 cache is fully utilized, and the running speed of these subroutines is improved by $35.3 \%$.

In the current implementation, as in the original mpiPOM code, the three-dimensional arrays of variables are stored sequentially as east-west $(x)$, north-south $(y)$, and vertical $(z)$, i.e. $i, j, k$ ordering. Two-dimensional arrays are stored in $i, j$ ordering. The vertical diffusion is solved using a tridiagonal solver that is calculated sequentially in the $z$ direction. For simplicity, in our kernel functions the grid is divided along $x$ and $y$. Each GPU thread then specifies an $(x, y)$ point in the horizontal direction and performs all of the calculations from the surface to the bottom. The thread blocks are divided as $32 \times 4$ subdomains in the $x-y$ plane. In the $x$ direction, the block number must be a multiple of 32 threads to perform consecutive and aligned memory access within a warp (NVIDIA, 2015). In the $y$ direction, we tested many thread numbers, such as 4 and 8 , and obtained similar performances. We ultimately choose 4 because this value produced more blocks and allowed us to distribute the workload more uniformly amongst the SMs. In addition, $128(=32 \times 4)$ threads are enough to maintain the full occupancy, which is the number of active threads in each multiprocessor.

In GPU computing, one is free to choose which arrays will be stored in an on-chip cache. Our experience involves putting the data along the horizontal direction into the readonly cache to reuse among threads, and putting the data along with vertical direction into the local memory for reuse within one thread.

Furthermore, we improve the global memory bandwidth by disabling the Error Checking and memory Correcting (ECC-off), as well as enhancing the clock on the GPU (GPU boost). This method improves the performance of the POM.gpu by $13.8 \%$.

\subsubsection{Results of the computational optimizations}

We divide all of the POM.gpu subroutines into three categories based on their different computational patterns. As shown in Table 2, in the POM.gpu, we deploy different opti- 


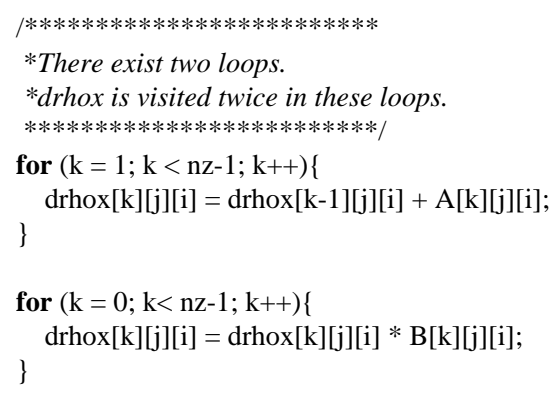

(a)Original CUDA-C code

Figure 4. A simple example of loop fusion.

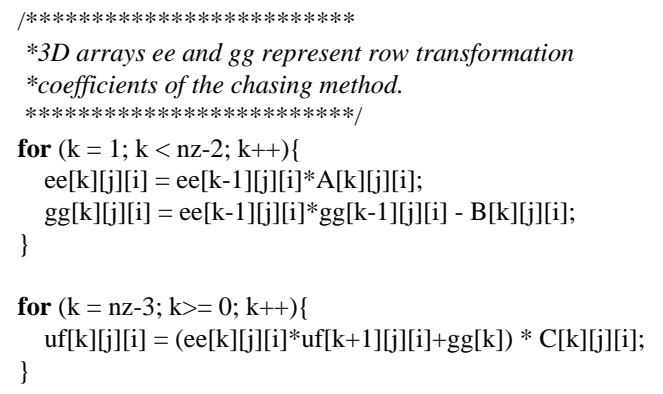

\section{(a)Original CUDA-C code}

Figure 5. A simple example of local memory blocking.

mizations in these categories to improve the performance of POM.gpu; these categories are described as follows.

1. Category 1: advection and horizontal diffusion $(a d v)$

This category has six subroutines, and calculates the advection, horizontal diffusion and the pressure gradient and Coriolis terms in the case of velocity. Here, it is possible to reuse data among adjacent threads, and the subroutines therefore benefit from using the read-only data cache. At the same time, the variables are calculated in different loops or in different functions such that the loop fusion and function fusion optimizations are applied to this part as well.

2. Category 2: vertical diffusion (ver)

This category has four subroutines and calculates the vertical diffusion. In this part, the chasing method is used in the tridiagonal solver in the $k$ direction. The main feature is that the data are accessed twice within one thread, once from the surface to the bottom and again from the bottom to the surface. The subroutines are significantly sped up after grouping the $k$-direction variable in the local memories.

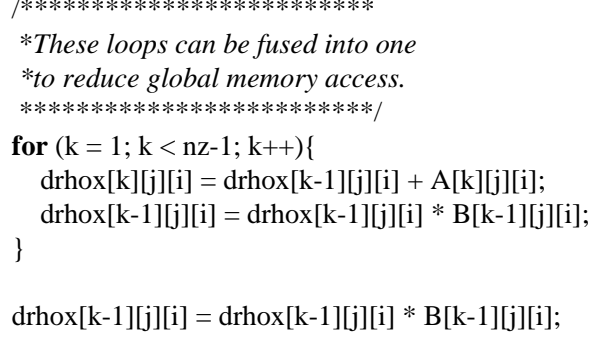

(b)Optimized CUDA-C code

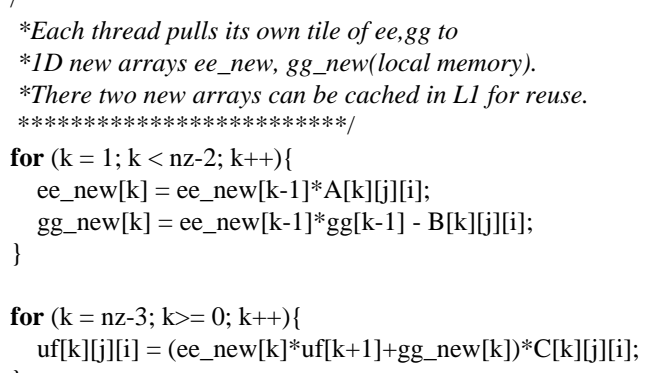

\section{(b)Optimized CUDA-C code}

3. Category 3: vorticity (vort), baroclinicity (baro), continuity equation (cont) and equation of state (state)

This category is less time-consuming than the two categories described above, but it also benefits from our optimizations. Because data reuse exists among threads, the use of a read-only data cache improves data locality. For the vort subroutine, there is data reuse within one thread, and thus the loop fusion improves the data locality.

\subsection{Communication optimizations among multiple GPUs}

In this section, we present the optimizing strategies for multiple GPUs. In the mpiPOM, the entire domain is split along the horizontal directions and each MPI process is responsible for the model's computation of one subdomain, following Jordi and Wang (2012). In the POM.gpu, we attach one MPI process to one GPU and move the complete computation to the GPU. The MPI process is in charge of the computation within each subdomain and of the data transfer between the GPU and the main memory. The data transfer between subdomains is handled by the GPUs directly. Shimokawabe et al. 
Table 2. Different subroutines adopt different optimizations in the POM.gpu.

\begin{tabular}{lcccccc}
\hline Subroutines & $\begin{array}{c}\text { Loop } \\
\text { fusion }\end{array}$ & $\begin{array}{c}\text { Function } \\
\text { fusion }\end{array}$ & $\begin{array}{c}\text { Read-only } \\
\text { data cache }\end{array}$ & $\begin{array}{c}\text { Local memory } \\
\text { blocking }\end{array}$ & $\begin{array}{c}\text { ECC-off and } \\
\text { GPU boost }\end{array}$ & Speedup \\
\hline Adv. and hor. diff. & $\sqrt{ }$ & $\sqrt{ }$ & $\sqrt{ }$ & & $\sqrt{ }$ & $2.05 \mathrm{X}$ \\
Ver. diff. & $\sqrt{ }$ & $\sqrt{ }$ & & $\sqrt{ }$ & $\sqrt{ }$ & $2.82 \mathrm{X}$ \\
Baroclinicity & $\sqrt{ }$ & & $\sqrt{ }$ & & $\sqrt{ }$ & $2.08 \mathrm{X}$ \\
Continuity equation & & & $\sqrt{ }$ & & $\sqrt{ }$ & $1.39 \mathrm{X}$ \\
Vorticity & $\sqrt{ }$ & & $\sqrt{ }$ & & $\sqrt{ }$ & $3.19 \mathrm{X}$ \\
State equation & & & $\sqrt{ }$ & & $1.35 \mathrm{X}$ \\
\hline
\end{tabular}

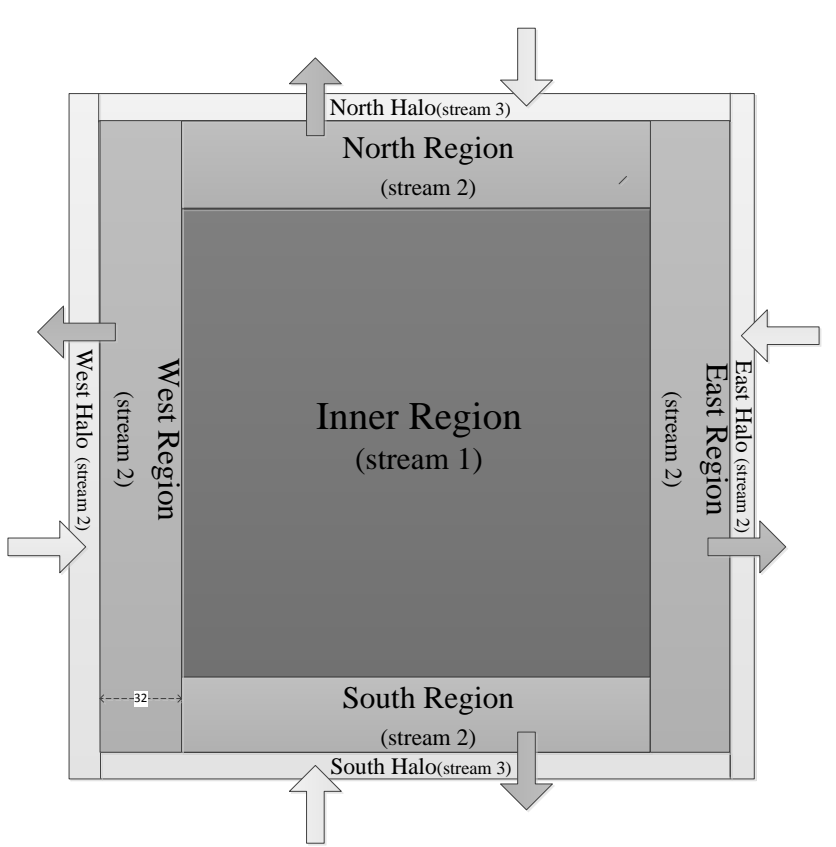

Figure 6. Data decomposition in the POM.gpu.

(2010) and Yang et al. (2013) proposed fine-grained overlapping methods of GPU computation and CPU communication to improve the computing performance. An important issue in their work is that the communications between multiple GPUs explicitly require the participation of the CPU. In our current work, we simply bypass the CPU in implementing the communication to fully exploit the capability of the GPUs.

At present, two MPI libraries, OpenMPI and MVAPICH2, provide support for the direct communication from the GPU to the main memory. This capability is referred to as CUDAaware MPI. We attempted to use MVAPICH2 to implement direct communication among multiple GPUs. However, we found that inter-domain communication occupied nearly $18 \%$ of the total runtime.

Instead, to fully overlap the boundary operations and MPI communications with computation, we adopt the data decomposition method shown in Fig. 6. The data region is decomposed into three regions: the inner region, the outer region, and a halo region which exchanges data with its neigh- bours. In our design, the inner region, which is the most timeconsuming, is allocated to stream 1 . The East/West outer region is allocated to stream 2 and the North/South outer region is allocated to stream 3. In the East/West outer region, the width is set to 32 to ensure consecutive and aligned memory access in a warp. All of the halo regions are also allocated to stream 2.

The workflow of multiple streams on the GPU is shown in Fig. 7. The East, West, North and South regions are common kernel functions that can run in parallel with the inner region through different streams. The communication operations between domains are implemented by an asynchronous CUDA memory copy. The corresponding synchronization operations between the CPU and the GPU or between the MPI processes are implemented by a synchronization CUDA function and a MPI barrier function. To overlap the subsequent communication with the inner region, stream 2 and stream 3 for the outer region have higher priority in preempting the computing resource from stream 1 at any time. Based on this workflow, the inter-domain communication is overlapped with the computation. The experimental results show that our design can remove the communication overhead taken by MVAPICH2.

\subsection{I / O optimizations between the GPUs and the CPUs}

The time consumed for $\mathrm{I} / \mathrm{O}$ in the mpiPOM is not significant. However, after we fully accelerate the model by GPU, it accounts for approximately $30 \%$ of the total runtime. The computing phase and the I / $\mathrm{O}$ phase are serial, which means that the GPU will remain idle until the CPU finishes the I/ O operations. Motivated by previous work on I / O overlapping (Huang et al., 2014), we designed a similar method following computations on a GPU and I / O operations on a CPU to run in parallel.

In the POM.gpu, we chose to launch more MPI processes. The MPI processes are divided into computing processes and I / O processes with different MPI communicators. The computing processes are responsible for launching kernel functions as usual, and the I / O processes are responsible for output. One I / O process attaches to one computing process and these two processes set their contexts on the same GPU. 


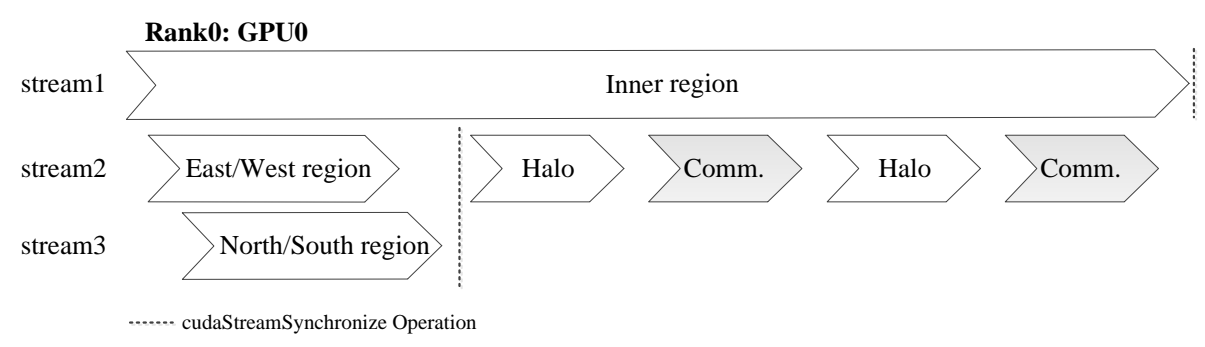

Figure 7. The workflow of multiple streams on the GPU. The Inner/East/West/North/South regions and Halo refer to the computation and update of the corresponding region. Comm. refers to the communication between processes, which implies synchronization.

Because the I / O processes must fetch data from the GPU, communication is necessary between them. The I/O processes obtain the device buffer pointers from the computing processes during the initialization phase. When writing history files, the computing processes are blocked and remain idle for a short time, waiting for I / O processes to fetch data. Then, the computing processes continue their computation, and the I/O processes complete their output in the background, as illustrated in Fig. 8. This method can be further optimized by placing the archive data in a set-aside buffer and carrying on the main calculation. However, the method requires more memory, which is not abundant in current K20X GPUs.

The advantage of this method is that it overlaps the I/O on the CPU with the model calculation on the GPU. In serial I / O, the GPU computing processes are blocked while data are sent to the CPU and written to disk. In overlapping I / O, the computing processes only wait for the data to be sent to the host. The bandwidth of data brought to the host is approximately $6 \mathrm{~GB} \mathrm{~s}^{-1}$, but the output bandwidth to the disk is approximately $100 \mathrm{MB} \mathrm{s}^{-1}$, as determined by the speed of the disk. Therefore, the overlapping method significantly accelerates the entire application.

\section{Experiments}

In this section, we first describe the specification of our platform and comparison methodology to validate the correctness of the POM.gpu. Furthermore, we present the performance and scalability of the POM.gpu compared with the mpiPOM.

\subsection{Platform setup}

The POM.gpu runs in a workstation consisting of two CPUs and four GPUs. The CPUs are $2.6 \mathrm{GHz} 8$-core Intel SandyBridge E5-2670. The GPUs are Nvidia Tesla K20X. The operating system is RedHat Enterprise Linux $6.3 \times 86 \_64$. All programs are complied with Intel compiler v14.0.1, CUDA 5.5 Toolkit, Intel MPI Library v4.1.3 and MVAPICH2 v1.9.

For comparison, the mpiPOM runs on the Tansuo 100 cluster at Tsinghua University consisting of 740 nodes. Each node is equipped with two $2.93 \mathrm{GHz}$ 6-core Intel Xeon X5670 CPUs and $32 \mathrm{~GB}$ of memory. The nodes are connected through an InfiniBand network. The operating system is RedHat Enterprise Linux $5.5 \times 86 \_64$. Programs on this platform are compiled with Intel compiler v11.1 and Intel MPI v4.0.2. The mpiPOM code is compiled with its original compiler flags, i.e. "-O3 -fp-model precise".

\subsection{The test case and the verification of accuracy}

The "dam-break" simulation (Oey, 2014) is conducted to verify the correctness and test the performance and scalability of the POM.gpu. It is a baroclinic instability problem that simulates flows produced by horizontal temperature gradients. The model domain is configured as a straight channel with a uniform depth of $50 \mathrm{~m}$. Periodic boundary conditions are used in the east-west direction, and the channel is closed in the north and south. Its horizontal resolution is $1 \mathrm{~km} \times 1 \mathrm{~km}$. The domain size of this test case is $962 \times 722$ horizontal grid points and 51 vertical sigma levels, which is limited by the capacity of one's GPU memory. Initially, the temperature in the southern half of the channel is 15 and $25^{\circ} \mathrm{C}$ in the northern half. The salinity is fixed at $35 \mathrm{psu}$. The fluid is then allowed to adjust. In the first 3-5 days, geostrophic adjustments occur. Then, an unstable wave develops due to baroclinic instability. Eventually, eddies are generated. Figure 9 shows the sea-surface height, sea-surface temperature (SST), and currents after 39 days. The scales of the frontal wave and eddies are determined by the Rossby radius of deformation. This dam-break case uses a single-precision format.

To verify the accuracy, we check the binary output files of the mpiPOM and the POM.gpu, as in Mak et al. (2011). The test results demonstrate that the variables velocity, temperature, salinity and sea-surface height are all identical.

\subsection{Model performance}

To understand the advantages of the optimizations in Sect. 4, we conducted different tests. The metrics of seconds per simulation day are measured to compare the model performance. 


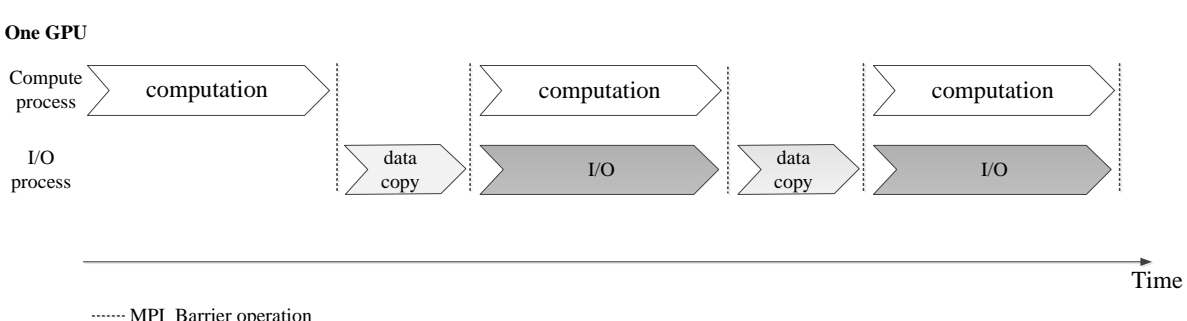

Figure 8. One computing process and one I / O process both set their contexts on the same GPU. During the data copy phase, the computing process remains idle and the I / O process will copy data from the GPU to the CPU through the cudaMemcpy function.
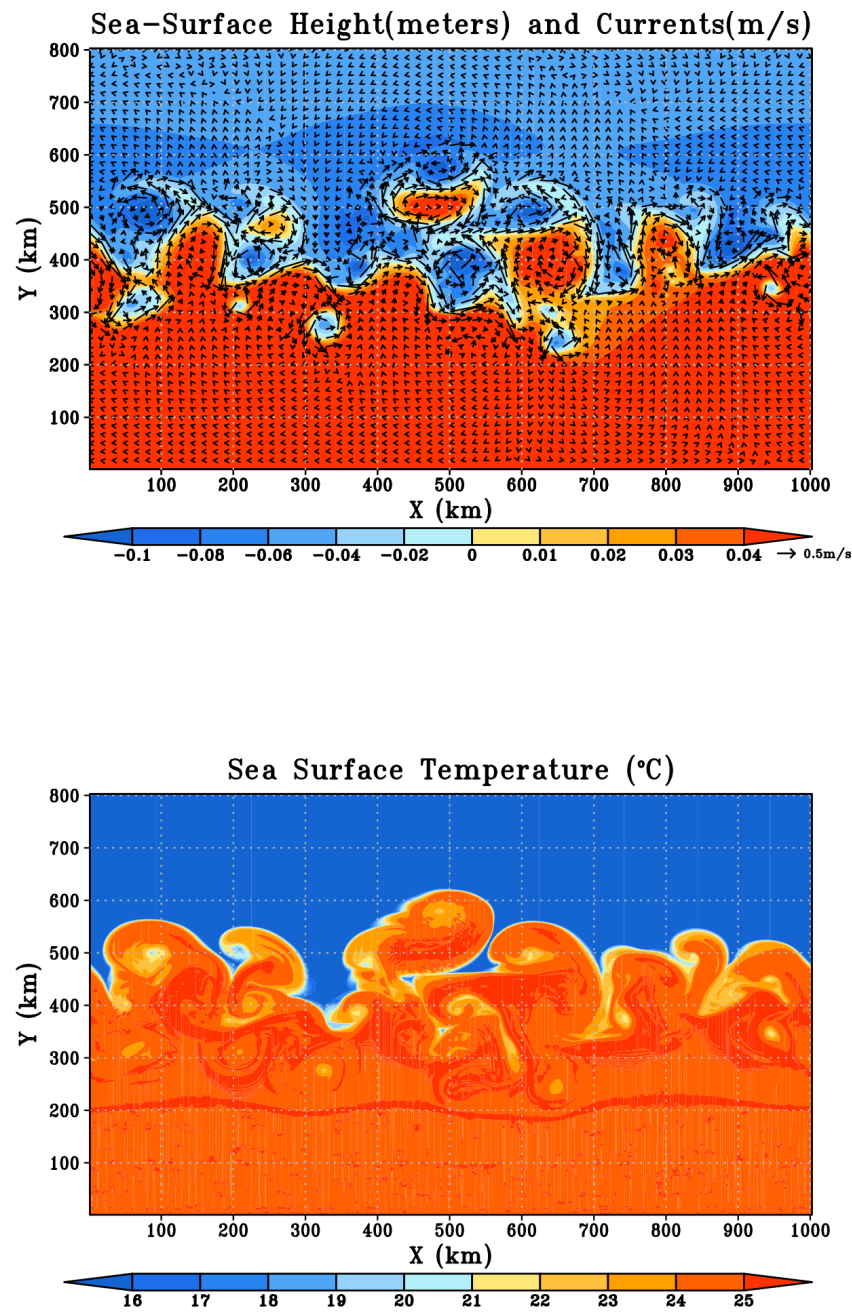

Figure 9. The model results after 39 days of simulation. For the top figure, the colour shading is the sea-surface height ( $\mathrm{SSH}$ ), and vectors are ocean currents. For the bottom figure, the colour shading is the sea-surface temperature (SST). Several warm and cold eddies are generated in the middle of the domain where the SST gradient is largest; their scales are determined by the Rossby radius of deformation.

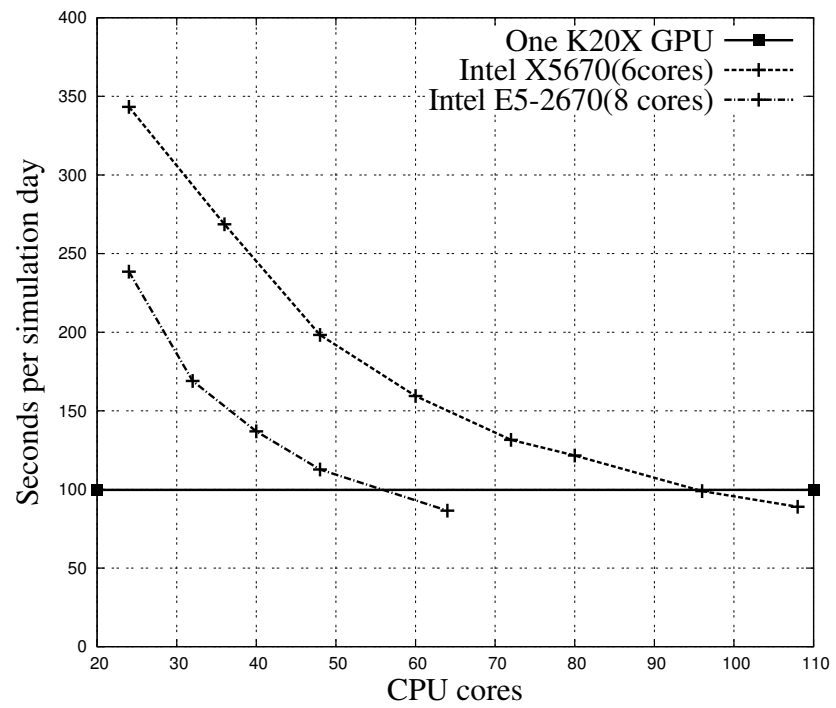

Figure 10. Performance comparison with different hardware platforms.

\subsubsection{Single GPU performance}

In our first test, we compare the performance of the mpiPOM using two different CPUs, the Intel X5670 CPU (six cores) and the Intel E5-2670 CPU (eight cores), with that obtained from the POM.gpu using one single GPU. Figure 10 shows that one K20X GPU can compete with approximately 55 E5$2670 \mathrm{CPU}$ cores to $95 \mathrm{X} 5670 \mathrm{CPU}$ cores in the simulation. From the parameters of the Intel E5-2670 CPU and Nvidia K20X GPU, we find that the ratio of memory bandwidth and the ratio of floating points performance are approximately $1: 5$ and $1: 10$, respectively. This means, if an application is strictly memory bandwidth limited, one GPU can compete with 5 CPUs; if an application is strictly computation limited, it can compete with 10 CPUs. Since the mpiPOM is memory bandwidth limited, the POM.gpu should provide an equivalent performance to the mpiPOM running on up to $5 \times 8=40$ CPU cores. Our procedure attempts to optimize memory access and we can further increase this number to 55 . 


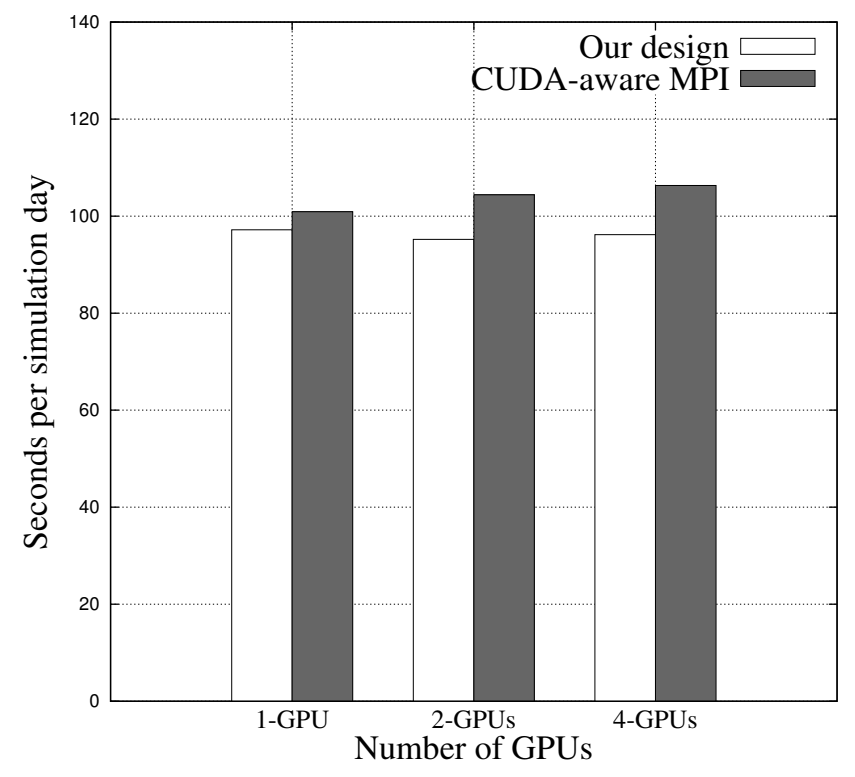

Figure 11. The weak scaling test between our communication overlapping method and the MVAPICH2 subroutines.

Table 3. The strong scaling result of POM.gpu.

\begin{tabular}{cccc}
\hline Number of GPUs & 1-GPU & 2-GPUs & 4-GPUs \\
\hline Time (s) & 97.2 & 48.7 & 26.3 \\
Efficiency & $100 \%$ & $99 \%$ & $92 \%$ \\
\hline
\end{tabular}

\subsubsection{Multiple GPU performance}

In the second test, we compare our communication overlapping method with the MVAPICH2 library. Figure 11 presents the weak scaling performance on multiple GPUs, where the grid size for each GPU is kept at $962 \times 722 \times 51$. When four GPUs are used with MVAPICH2, approximately $18 \%$ of the total runtime is consumed by inter-domain communication and boundary operations. This overhead can be greatly reduced by our communication overlapping method.

In the third test, we fix the global grid size at $962 \times 722 \times$ 51, and measure the strong scaling performance of POM.gpu. Table 3 shows that the strong scaling efficiency is $99 \%$ on two GPUs and $92 \%$ on four GPUs. When more GPUs are used, the size of each subdomain becomes smaller. This decreases the performance of POM.gpu in two aspects. First, the communication overhead may exceed the computation time of the inner region as the size of each subdomain decreases. As a result, the overlapping methods in Sect. 4.2 are not effective. Second, there are many "small" kernels in the POM.gpu code, in which the calculation is simple and less time-consuming. With fewer inner region computations, the overhead of kernel launching and implicit synchronization with kernel execution must be counted.

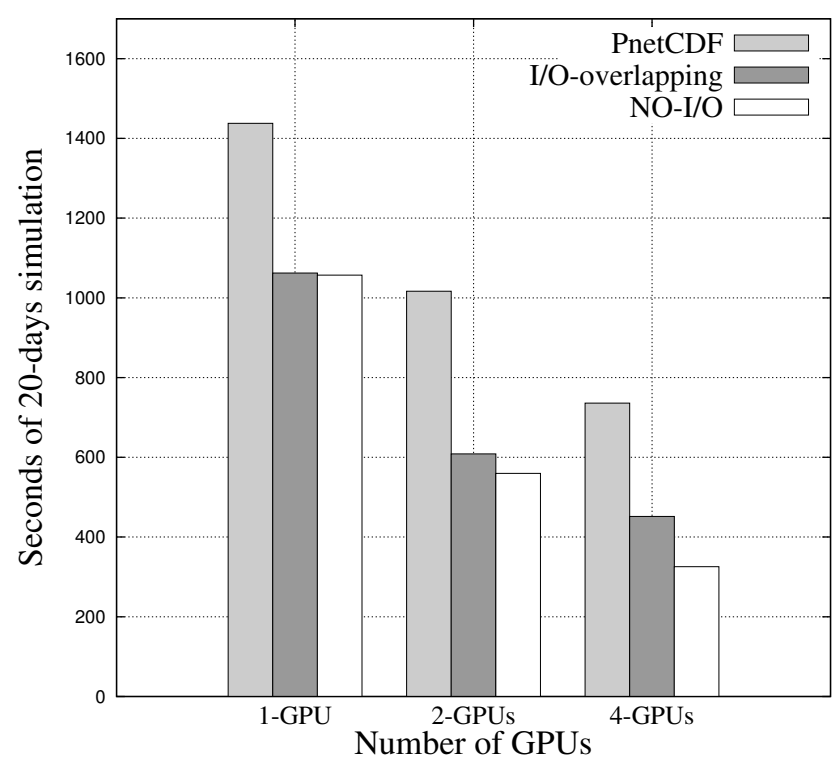

Figure 12. I / O test for the POM.gpu.

\subsubsection{I / O performance}

In the fourth test, we compare our I / O overlapping method with the parallel NetCDF (PnetCDF) method and NO-I / O. NO-I / O means that all I / O operations are disabled in the program and that the time measured is the pure computing time. This simulation is run for 20 days, and the history files are output daily. The final history files in NetCDF format are approximately 12 GB. Figure 12 shows that the I / O overlapping method outperforms the PnetCDF method. For one and two GPUs, the overall runtime decreases from 1694/1142 to $1239 / 688 \mathrm{~s}$, which is close to the NO-I / O. The extra overhead of our method compared with NO-I / O involves the computing processes that need to be blocked until the I/O processes obtain data from the GPUs. When running with four GPUs, the output time exceeds the computation time. Then, the I/O phase cannot be fully overlapped with the model computation phase. The overall runtime equals the sum of the computation time and the non-overlapped I/ O time.

\subsubsection{Comparison with a cluster}

In the last test, we compare the performance of POM.gpu on a workstation containing four GPUs with that on the Tansuo100 cluster. Three different high-resolution grids (Grid-1: $962 \times 722 \times 51$; Grid-2: $1922 \times 722 \times 51$; Grid-3: $1922 \times 1442 \times 51)$ are used. Figure 13 shows that our workstation with four GPUs is comparable to 408 standard CPU cores $(=34$ nodes $\times 12$ cores/node) in the simulation. Because the thermal design power of one X5670 CPU is $95 \mathrm{~W}$ and that of one K20X GPU is $235 \mathrm{~W}$, we reduce the energy consumption by a factor of 6.8 . Theoretically, as the subdo- 


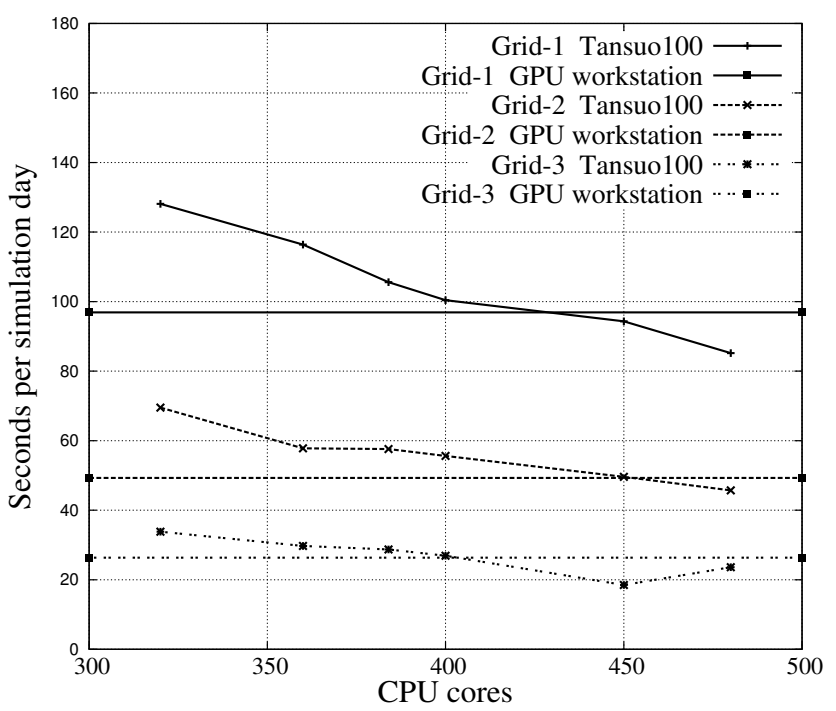

Figure 13. Performance test of four GPUs compared with the Tansuo100 cluster.

main of each MPI process becomes smaller, the cache hit ratio of the mpiPOM code will increase. This will greatly alleviate the memory bandwidth-limited problem. However, in the simulation on 408 standard CPU cores, the MPI communication may occupy more than $40 \%$ of the total execution time. When scaling to over 450 cores, the mpiPOM simulation may instead become slower, as shown in Fig. 13. Therefore, for high-resolution ocean modelling, our POM.gpu has a clear advantage compared to the original mpiPOM.

\section{Code availability}

The POM.gpu version 1.0 is available at https:/github.com/ hxmhuang/POM.gpu. To reproduce the test case in Sect. 5, the run_exp002.sh script is provided to compile and execute the POM.gpu code.

\section{Conclusions and future work}

In this paper, we develop POM.gpu, a full GPU solution based on the mpiPOM. Unlike previous GPU porting, the POM.gpu code distributes the model computations on the GPU. Our main contributions include optimizing the code on each of the GPUs, the communications between GPUs, and the I/ O process between the GPUs and the CPUs. Using a workstation with four GPUs, we achieve the performance of a powerful CPU cluster with 408 standard CPU cores. Our model also reduces the energy consumption by a factor of 6.8. It is a cost-effective and energy-efficient strategy for high-resolution ocean modelling. We have described the method and tests in detail and, with the availability of the POM.gpu code, our experiences may hopefully be useful to developers and designers of other general circulation models.
In our current POM.gpu, we design a large number of kernel functions because we port the entire mpiPOM one subroutine at a time. This was done to simplify the debugging of POM.gpu and to check that the results are consistent with the mpiPOM. In our future work, we will adjust the code structure of POM.gpu and adopt aggressive function fusion to further improve the performance.

Previous studies proposed to take advantage of data locality between time steps by time skewing (McCalpin and Wonnacott, 1999; Wonnacott, 2000), thus transforming the problem of memory bandwidth into the problem of computation. However, the real-world ocean models, including the mpiPOM, often involve hundreds of thousands lines of code, and analysing the data dependency and applying time skewing in such a context are challenging and difficult. We leave that to the next-generation POM.gpu.

\section{The Supplement related to this article is available online at doi:10.5194/gmd-8-2815-2015-supplement.}

Acknowledgements. The author would like to thank David Webb, Robert Marsh and the anonymous reviewer for their valuable comments and improvements regarding the presentation of this manuscript. This study was supported by funding from the National Natural Science Foundation of China (41375102), the National Grand Fundamental Research 973 Program of China (no. 2014CB347800), and the National High Technology Development Program of China (2011AA01A203).

Edited by: R. Marsh

\section{References}

Allen, J. S. and Newberger, P. A.: Downwelling Circulation on the Oregon Continental Shelf. Part I: Response to Idealized Forcing, J. Phys. Oceanogr., 26, 2011-2035, doi:10.1175/15200485(1996)026<2011:DCOTOC>2.0.CO;2, 1996.

Berntsen, J. and Oey, L.-Y.: Estimation of the internal pressure gradient in $\sigma$-coordinate ocean models: comparison of second, fourth-, and sixth-order schemes, Ocean Dynam., 60, 317-330, 2010.

Blumberg, A. F. and Mellor, G. L.: Diagnostic and prognostic numerical circulation studies of the South Atlantic Bight, J. Geophys. Res.-Oceans, (1978-2012), 88, 4579-4592, 1983.

Blumberg, A. F. and Mellor, G. L.: A description of a threedimensional coastal ocean circulation model, Coast. Est. Sci., 4 1-16, 1987.

Browne, S., Dongarra, J., Garner, N., Ho, G., and Mucci, P.: A portable programming interface for performance evaluation on modern processors, Int. J. High Perf. Comp. Appl., 14, 189-204, 2000.

Chang, Y.-L. and Oey, L.-Y.: Instability of the North Pacific subtropical countercurrent, J. Phys. Oceanogr., 44, 818-833, 2014. 
Chapman, B., Jost, G., and Van Der Pas, R.: Using OpenMP: portable shared memory parallel programming, vol. 10, The MIT Press, 2008.

Ezer, T. and Mellor, G. L.: A numerical study of the variability and the separation of the Gulf Stream, induced by surface atmospheric forcing and lateral boundary flows, J. Phys. Oceanogr., 22, 660-682, 1992.

Gopalakrishnan, S., Liu, Q., Marchok, T., Sheinin, D., Surgi, N., Tuleya, R., Yablonsky, R., and Zhang, X.: Hurricane Weather Research and Forecasting (HWRF) model scientific documentation, edited by: Bernardet, L., 75, 2010.

Gopalakrishnan, S., Liu, Q., Marchok, T., Sheinin, D., Surgi, N., Tong, M., Tallapragada, V., Tuleya, R., Yablonsky, R., and Zhang, X.: Hurricane Weather Research and Forecasting (HWRF) model: 2011 scientific documentation, edited by: Bernardet, L., 2011.

Govett, M., Middlecoff, J., and Henderson, T.: Running the NIM next-generation weather model on GPUs, in: Cluster, Cloud and Grid Computing (CCGrid), 2010 10th IEEE/ACM International Conference on, 792-796, IEEE, 2010.

Gropp, W. D., Lusk, E. L., and Thakur, R.: Using MPI-2: Advanced features of the message-passing interface, vol. 2, Globe Pequot, 1999.

Guo, X., Miyazawa, Y., and Yamagata, T.: The Kuroshio Onshore Intrusion along the Shelf Break of the East China Sea: The Origin of the Tsushima Warm Current, J. Phys. Oceanogr., 36, 2006.

Henderson, T., Middlecoff, J., Rosinski, J., Govett, M., and Madden, P.: Experience applying Fortran GPU compilers to numerical weather prediction, in: Application Accelerators in HighPerformance Computing (SAAHPC), 2011 Symposium, 34-41, IEEE, 2011.

Huang, S.-M. and Oey, L.: Right-side cooling and phytoplankton bloom in the wake of a tropical cyclone, J. Geophys. Res.Oceans, 2015.

Huang, X. M., Wang, W. C., Fu, H. H., Yang, G. W., Wang, B., and Zhang, C.: A fast input/output library for high-resolution climate models, Geosci. Model Dev., 7, 93-103, doi:10.5194/gmd-7-932014, 2014.

Isobe, A., Kako, S., Guo, X., and Takeoka, H.: Ensemble numerical forecasts of the sporadic Kuroshio water intrusion (kyucho) into shelf and coastal waters, Ocean Dyn., 62, 633-644, 2012.

Jordi, A. and Wang, D.-P.: sbPOM: A parallel implementation of Princenton Ocean Model, Environ. Model. Softw., 38, 59-61, 2012.

Kagimoto, T. and Yamagata, T.: Seasonal transport variations of the Kuroshio: An OGCM simulation, J. Phys. Oceanogr., 27, 403418, 1997.

Korres, G., Hoteit, I., and Triantafyllou, G.: Data assimilation into a Princeton Ocean Model of the Mediterranean Sea using advanced Kalman filters, J. Marine Syst., 65, 84-104, 2007.

Kurihara, Y., Bender, M. A., Tuleya, R. E., and Ross, R. J.: Improvements in the GFDL hurricane prediction system, Mon. Weather Rev., 123, 2791-2801, 1995.

Kurihara, Y., Tuleya, R. E., and Bender, M. A.: The GFDL hurricane prediction system and its performance in the 1995 hurricane season., Mon. Weather Rev., 126, 1306-1322, 1998.

Leutwyler, D., Fuhrer, O., Cumming, B., Lapillonne, X., Gysi, T., Lüthi, D., Osuna, C., and Schär, C.: Towards Cloud-Resolving European-Scale Climate Simulations using a fully GPU-enabled
Prototype of the COSMO Regional Model, in: EGU General Assembly Conference Abstracts, vol. 16, p. 11914, 2014.

Lin, X., Xie, S.-P., Chen, X., and Xu, L.: A well-mixed warm water column in the central Bohai Sea in summer: Effects of tidal and surface wave mixing, J. Geophys. Res.-Oceans, 111, C11017, doi:10.1029/2006JC003504, 2006.

Linford, J. C., Michalakes, J., Vachharajani, M., and Sandu, A.: Multi-core acceleration of chemical kinetics for simulation and prediction, in: Proceedings of the Conference on High Performance Computing Networking, Storage and Analysis, p. 7, ACM, 2009.

Mak, J., Choboter, P., and Lupo, C.: Numerical ocean modeling and simulation with CUDA, in: OCEANS 2011, 1-6, IEEE, 2011.

McCalpin, J. and Wonnacott, D.: Time skewing: A value-based approach to optimizing for memory locality, Tech. rep., Technical Report DCS-TR-379, Department of Computer Science, Rugers University, 477-480, 1999.

Michalakes, J. and Vachharajani, M.: GPU acceleration of numerical weather prediction, Parallel Proc. Lett., 18, 531-548, 2008.

Miyazawa, Y., Zhang, R., Guo, X., Tamura, H., Ambe, D., Lee, J.S., Okuno, A., Yoshinari, H., Setou, T., and Komatsu, K.: Water mass variability in the western North Pacific detected in a 15year eddy resolving ocean reanalysis, J. Oceanogr., 65, 737-756, 2009.

Newberger, P. and Allen, J. S.: Forcing a three-dimensional, hydrostatic, primitive-equation model for application in the surf zone: 1. Formulation, J. Geophys. Res.-Oceans, (1978-2012), 112, 2007a.

Newberger, P. A. and Allen, J. S.: Forcing a three-dimensional, hydrostatic, primitive-equation model for application in the surf zone: 2. Application to DUCK94, J. Geophys. Res.-Oceans, 112, 2007b.

NVIDIA: CUDA C Best Practices Guide, available at: http://docs.nvidia.com/cuda/cuda-c-best-practices-guide/ index.html\#coalesce\%d-access-to-global-memory (last access: April 2015), 2015.

Oey, L., Chang, Y.-L., Lin, Y.-C., Chang, M.-C., Xu, F.-H., and Lu, H.-F.: ATOP-the Advanced Taiwan Ocean Prediction System based on the mpiPOM Part 1: model descriptions, analyses and results, Terr Atmos Ocean Sci, 24, 2013.

Oey, L.-Y.: A wetting and drying scheme for POM, Ocean Modelling, 9, 133-150, 2005.

Oey, L.-Y.: Geophysical Fluid Modeling with the mpi version of the Princeton Ocean Model (mpiPOM). Lecture Notes, 70 pp., ftp://profs.princeton.edu/leo/lecture-notes/ OceanAtmosModeling/Notes/GFModellingUsingMpiPOM.pdf (last access: January 2014), 2014.

Oey, L.-Y. and Chen, P.: A model simulation of circulation in the northeast Atlantic shelves and seas, J. Geophys. Res.-Oceans, 97, 20087-20115, 1992a.

Oey, L.-Y. and Chen, P.: A nested-grid ocean model: With application to the simulation of meanders and eddies in the Norwegian Coastal Current, J. Geophys. Res.-Oceans, (1978-2012), 97, 20 063-20 086, 1992b.

Oey, L.-Y., Mellor, G. L., and Hires, R. I.: A three-dimensional simulation of the Hudson-Raritan estuary. Part I: Description of the model and model simulations, J. Phys. Oceanogr., 15, 1676$1692,1985 \mathrm{a}$. 
Oey, L.-Y., Mellor, G. L., and Hires, R. I.: A three-dimensional simulation of the Hudson-Raritan estuary. Part II: Comparison with observation, J. Phys. Oceanogr., 15, 1693-1709, 1985 b.

Oey, L.-Y., Mellor, G. L., and Hires, R. I.: A three-dimensional simulation of the Hudson-Raritan estuary. Part III: Salt flux analyses, J. Phys. Oceanogr., 15, 1711-1720, 1985c.

Oey, L.-Y., Lee, H.-C., and Schmitz, W. J.: Effects of winds and Caribbean eddies on the frequency of Loop Current eddy shedding: A numerical model study, J. Geophys. Res.-Oceans, 108, 3324, doi:10.1029/2002JC001698, 2003.

Shimokawabe, T., Aoki, T., Muroi, C., Ishida, J., Kawano, K., Endo, T., Nukada, A., Maruyama, N., and Matsuoka, S.: Proceedings of the International Conference for High Performance Computing, Networking, Storage and Analysis, 1-11, An 80-fold speedup, 15.0 TFlops full GPU acceleration of non-hydrostatic weather model ASUCA production code, 2010.

Siewertsen, E., Piwonski, J., and Slawig, T.: Porting marine ecosystem model spin-up using transport matrices to GPUs, Geosci. Model Dev., 6, 17-28, doi:10.5194/gmd-6-17-2013, 2013.

Smolarkiewicz, P. K.: A fully multidimensional positive definite advection transport algorithm with small implicit diffusion, J. Comp. Phys., 54, 325-362, 1984.

Sun, J., Oey, L., Xu, F., Lin, Y., Huang, S., and Chang, R.: The Influence of Ocean on Typhoon Nuri (2008), in: AGU Fall Meeting Abstr., 1, L3360, available at: http://adsabs.harvard.edu/abs/ 2014AGUFM.A33L3360S, 2014.

Sun, J., Oey, L.-Y., Chang, R., Xu, F., and Huang, S.-M.: Ocean response to typhoon Nuri (2008) in western Pacific and South China Sea, Ocean Dynam., 65, 735-749, 2015.

Varlamov, S. M., Guo, X., Miyama, T., Ichikawa, K., Waseda, T., and Miyazawa, Y.: M2 baroclinic tide variability modulated by the ocean circulation south of Japan, J. Geophys. Res.-Oceans, 2015 .
Wonnacott, D.: Using time skewing to eliminate idle time due to memory bandwidth and network limitations, in: Parallel and Distributed Processing Symposium, 2000. IPDPS 2000, Proceedings, 14th International, 171-180, IEEE, 2000.

$\mathrm{Xu}$, F.-H. and Oey, L.-Y.: The origin of along-shelf pressure gradient in the Middle Atlantic Bight, J. Phys. Oceanogr., 41, 1720 1740, 2011.

$\mathrm{Xu}$, F.-H. and Oey, L.-Y.: State analysis using the Local Ensemble Transform Kalman Filter (LETKF) and the three-layer circulation structure of the Luzon Strait and the South China Sea, Ocean Dynam., 64, 905-923, 2014.

$\mathrm{Xu}$, F.-H. and Oey, L.-Y.: Seasonal SSH variability of the Northern South China Sea, J. Phys. Oceanogr., 45, 1595-1609, 2015.

Xu, F.-H., Oey, L.-Y., Miyazawa, Y., and Hamilton, P.: Hindcasts and forecasts of Loop Current and eddies in the Gulf of Mexico using local ensemble transform Kalman filter and optimuminterpolation assimilation schemes, Ocean Model., 69, 22-38, 2013.

Yang, C., Xue, W., Fu, H., Gan, L., Li, L., Xu, Y., Lu, Y., Sun, J., Yang, G., and Zheng, W.: A peta-scalable CPU-GPU algorithm for global atmospheric simulations, in: Proceedings of the 18th ACM SIGPLAN symposium on Principles and practice of parallel programming, 1-12, ACM, 2013.

Yin, X.-Q. and Oey, L.-Y.: Bred-ensemble ocean forecast of Loop Current and rings, Ocean Model., 17, 300-326, 2007.

Zavatarelli, M. and Mellor, G. L.: A numerical study of the Mediterranean Sea circulation, J. Phys. Oceanogr., 25, 1384-1414, 1995.

Zavatarelli, M. and Pinardi, N.: The Adriatic Sea modelling system: a nested approach, Ann. Geophys., 21, 345-364, 10.5194/ angeo-21-345-2003, 2003.

Zhenya, S., Haixing, L., Xiaoyan, L., and Zhao, W.: The Application of GPU in Ocean General Circulation Mode POP, Comp. Appl. Softw., 27, 27-29, 2010. 